\title{
Calculating repetitively
}

S.Gopinath Kamath* ${ }^{a}$

Department of Mathematics, Indian Institute of Technology Madras

Chennai 600 036, India

*e-mail: kamath@iitm.ac.in

Abstract:

The Antonsen - Bormann idea was originally proposed by these authors for the computation of the heat kernel in curved space; it was also used by the author recently with the same objective but for the Lagrangian density for a real massive scalar field in $2+1$ dimensional stationary curved space. Subsequently, it was reworked with advantage - but to determine the zeta function for the said model using the Schwinger operator expansion. The repetitive nature of that calculation at all higher orders $(\geq$ 3 ) in the gravitational constant G suggests the use of the Dirac delta-function and one of its integral representations - in that it is convenient to obtain answers; in anticipation of its systematic application to all orders $\geq 3$ in $\mathrm{G}$ and the exact evaluation of $\zeta(\mathrm{s})$ this paper illustrates in detail the evaluation of some integrals relevant to the third order calculation.

$36^{\text {th }}$ International Conference on High Energy Physics

July $4-11,2012$

Melbourne, Australia

${ }^{a}$ Speaker: S. Gopinath Kamath 


\section{Introduction}

The integral $I_{n}=\int_{0}^{\pi / 2} \sin ^{n} x d x$ with $n$ a non - negative integer is a textbook ${ }^{1}$ example of a repetitive calculation; thus, for $n>2$ one gets

$$
I_{n}=\frac{n-1}{n} I_{n-2}=\frac{n-1}{n} \frac{n-3}{n-2} I_{n-4}
$$

Continuing in this way one arrives at $I_{0}=\frac{\pi}{2}$ or $I_{1}=1$ depending on whether $n$ is even or odd; a well - known byproduct from eq.(1) being the Wallis formula ${ }^{1}$

$$
\frac{\pi}{2}=\lim _{m \rightarrow \infty}\left[\left\{\frac{2.4 .6 \ldots .2 m}{1.3 .5 \ldots .(2 m-1)}\right\}^{2} \frac{1}{2 m+1} \frac{I_{2 m}}{I_{2 m+1}}\right]
$$

with $\lim _{m \rightarrow \infty} \frac{I_{2 m}}{I_{2 m+1}}=1$.

We present another example of a repetitive calculation that we shall motivate later on in this paper. Much of this paper is an adjunct to an earlier version ${ }^{2}$ in that it presents the necessary steps to complete the third order calculation of the zeta-function discussed therein; being tentative and incomplete it warranted a second look and a reader-friendly exposition is given here. Parenthetically, the method presented here is substantial and was not used in Ref.2.

\section{Some integrals, their origin and evaluation}

Consider the integrals

$K_{0}(\vec{p})=\left(-\frac{\lambda}{4 \pi}\right)^{3}(-2)^{2} \int r_{1} \frac{\left(r_{2}-q_{2}\right)\left(r_{2} q_{1}-r_{1} q_{2}\right)}{(\vec{r}-\vec{q})^{2}} e^{-x r^{2}} \frac{\left(q_{2}-p_{2}\right)\left(q_{2} p_{1}-q_{1} p_{2}\right)}{(\vec{q}-\vec{p})^{2}} e^{-z q^{2}}$

$K_{1}(\vec{p})=\left(-\frac{\lambda}{4 \pi}\right)^{3}(-2)^{2} \int q_{1} \frac{\left(p_{2}-r_{2}\right)\left(p_{2} r_{1}-p_{1} r_{2}\right)}{(\vec{p}-\vec{r})^{2}} e^{-x r^{2}} \frac{\left(q_{2}-p_{2}\right)\left(q_{2} p_{1}-q_{1} p_{2}\right)}{(\vec{q}-\vec{p})^{2}} e^{-z q^{2}}$

$K_{2}(\vec{p})=\left(-\frac{\lambda}{4 \pi}\right)^{3}(-2)^{2} \int p_{1} \frac{\left(p_{2}-r_{2}\right)\left(p_{2} r_{1}-p_{1} r_{2}\right)}{(\vec{p}-\vec{r})^{2}} e^{-x r^{2}} \frac{\left(r_{2}-q_{2}\right)\left(r_{2} q_{1}-r_{1} q_{2}\right)}{(\vec{r}-\vec{q})^{2}} e^{-z q^{2}}$

$K_{3}(\vec{p})=\left(-\frac{\lambda}{4 \pi}\right)^{3}(-2)^{3} \int e^{-x r^{2}} \frac{\left(p_{2}-r_{2}\right)\left(p_{2} r_{1}-p_{1} r_{2}\right)}{(\vec{p}-\vec{r})^{2}} \frac{\left(r_{2}-q_{2}\right)\left(r_{2} q_{1}-r_{1} q_{2}\right)}{(\vec{r}-\vec{q})^{2}} \frac{\left(q_{2}-p_{2}\right)\left(q_{2} p_{1}-q_{1} p_{2}\right)}{(\vec{q}-\vec{p})^{2}} e^{-z} q^{2}$

and

$$
K_{4}(\vec{p})=\left(-\frac{\lambda}{4 \pi}\right)^{3}(-2) \int e^{-x r^{2}} \frac{\left(r_{2}-q_{2}\right)\left(r_{2} q_{1}-r_{1} q_{2}\right)}{(\vec{r}-\vec{q})^{2}} q_{1} p_{1} e^{-z q^{2}}
$$

with $\int \quad$ in each of the above being short hand for $\int d^{2} r d^{2} q, q^{2}=q_{1}^{2}+q_{2}^{2}, r^{2}=r_{1}^{2}+r_{2}^{2}$ and $x$ and $z$ being real and non-negative. Of these $K_{1}$ and $K_{4}$ are easily evaluated as

$K_{1}=-\frac{1}{\pi}\left(\frac{\lambda}{4}\right)^{3} p_{1} e^{-(x+z) \vec{p}^{2}} \frac{1}{b c^{2}}\left(-1+e^{x \vec{p}^{2}}\right)\left\{c\left(p_{2}^{2} e^{z \vec{p}^{2}}-p_{1}^{2}\right)-z\left(p_{1}^{2}-p_{2}^{2}\right)\left(1-e^{z \vec{p}^{2}}\right)\right\}$

$K_{4}=\frac{1}{2 \pi}\left(\frac{\lambda}{4}\right)^{3} p_{1} \frac{1}{x z(x+z)}$

with $c=z^{2} \vec{p}^{2}$ and $b=x^{2} \vec{p}^{2}$.The remaining integrals - especially $K_{3}$ - are tedious to evaluate thus begging an alternative; while deferring its details to the sequel it pays to briefly recall their origin here: 
They are obtained from the momentum space representation of the order $G^{3}$ term in the Schwinger operator expansion ${ }^{2,5,6}$ for $e^{-\left(p^{2}+H_{I}\right) t}$ namely,

$$
(-t)^{3} \int_{0}^{1} u^{2} d u \int_{0}^{1} u_{1} d u_{1} \int_{0}^{1} d u_{2}\left\{\begin{array}{c}
e^{-t(1-u) p^{2}} \int d^{2} r d^{2} s\left\langle p\left|H_{I}\right| r\right\rangle e^{-t u\left(1-u_{1}\right) r^{2}}\left\langle r\left|H_{I}\right| s\right\rangle \\
e^{-t u u_{1}\left(1-u_{2}\right) s^{2}}\left\langle s\left|H_{I}\right| p\right\rangle e^{-t u u_{1} u_{2} p^{2}}
\end{array}\right\}
$$

with the operator $H_{I}=-\frac{\lambda}{r^{4}}\left[\left(y^{2}-x^{2}\right) p_{1}-2 x y p_{2}\right], r^{2}=x^{2}+y^{2}, \lambda=4 G J, G$ being the gravitational constant and one of the matrix elements $\left\langle p\left|H_{I}\right| r\right\rangle$ for example being

$\left\langle p\left|H_{I}\right| r\right\rangle=-\frac{\lambda}{4 \pi}\left(r_{1}-2 \frac{\left(p_{2}-r_{2}\right)\left(p_{2} r_{1}-p_{1} r_{2}\right)}{(\vec{r}-\vec{p})^{2}}\right)$. Of the eight possible terms in (4) three are zero by symmetry leaving the five apparently nonzero terms given in eq.(2).The interested reader is referred to Refs. 2 and 4 for details.

Returning now to evaluation of the integrals in (2) we begin with $K_{0}$ written as

$K_{0}=\left(-\frac{\lambda}{4 \pi}\right)^{3}(-2)^{2} \int \delta(\vec{q}-\vec{s}) r_{1} \frac{\left(r_{2}-s_{2}\right)\left(r_{2} s_{1}-r_{1} s_{2}\right)}{(\vec{r}-\vec{s})^{2}} e^{-x r^{2}} \frac{\left(q_{2}-p_{2}\right)\left(q_{2} p_{1}-q_{1} p_{2}\right)}{(\vec{q}-\vec{p})^{2}} e^{-z q^{2}}$

with $\int \quad$ now being short hand for $\int d^{2} r d^{2} q d^{2} s$. The introduction of the Dirac delta-function in (5) is a point of departure in this paper - for on using the integral representation ${ }^{3}$

$$
\delta(\vec{q}-\vec{s})=\left(\frac{1}{2 \pi}\right)^{2} \iint_{-\infty}^{\infty} d \alpha d \beta e^{i \alpha\left(q_{1}-s_{1}\right)+i \beta\left(q_{2}-s_{2}\right)}
$$

one can now do the $s, q$ and $r$ integration in (5) easily. Parenthetically, the Dirac delta-function was also used elsewhere $^{4}$ but with the limit representation ${ }^{3}$

$$
\delta(x)=\lim _{\epsilon \rightarrow 0+} \frac{1}{2 \sqrt{\pi \epsilon}} e^{-x^{2} / 4 \epsilon}
$$

Eq.(7) however is ineffectual and is therefore given up in favour of (5) here; on doing the $r$ integration first one gets

$\frac{\pi}{2} e^{-x s^{2}} \int_{0}^{\infty} d \mu \frac{e^{\frac{a}{h}}}{h^{3}}\left(h s_{2}^{2}+\mu\left(s_{1}^{2}-s_{2}^{2}\right)\right)$

with $a=x^{2} s^{2}, h=\mu+x$; returning to (5) the $s$ integration can now be completed to get $\frac{\pi e^{-\frac{c}{b}}}{4 b^{3}}\left[h\left(2 b-\beta^{2}\right)+\mu\left(\beta^{2}-\alpha^{2}\right)\right]$ the answer after the two integrations being

$\left(\frac{\pi}{2}\right)^{2} \int_{0}^{\infty} d \mu \frac{e^{-\frac{c}{b}}}{2 b^{3} h^{3}}\left[h\left(2 b-\beta^{2}\right)+\mu\left(\beta^{2}-\alpha^{2}\right)\right]=\left(\frac{\pi}{2}\right)^{2} \frac{1}{2 c^{2} x^{3}} e^{-\frac{c}{x}}\left\{\frac{x}{2}\left(\alpha^{2}-\beta^{2}\right)-c \alpha^{2}\right\}$

with $h b=\mu x, 4 c \equiv \alpha^{2}+\beta^{2}$. It is prudent to complete the $\alpha, \beta$ integration now prior to the $q$ integration to get

$$
T \equiv \frac{\pi x}{8}\left(q_{2}^{2}-q_{1}^{2}\right) \int_{0}^{\infty} v d v \frac{e^{-\frac{q^{2}}{4 k}}}{k^{3}}-\frac{\pi}{4} \int_{0}^{\infty} d v \frac{e^{-\frac{q^{2}}{4 k}}}{k^{3}}\left(2 k-q_{1}^{2}\right)
$$




$$
=8 \pi\left\{\frac{x}{q^{2}}\left(q_{2}^{2}-q_{1}^{2}\right)+\frac{2\left(q_{2}^{2}-q_{1}^{2}\right)}{q^{4}}\left(-1+e^{-x q^{2}}\right)-\frac{2 x q_{1}^{2}}{q^{2}} e^{-x q^{2}}\right\} \equiv 8 \pi F
$$

Therefore

$$
K_{0}=-\frac{1}{\pi^{2}}\left(\frac{\lambda}{4 x}\right)^{3} \int F \frac{\left(q_{2}-p_{2}\right)\left(q_{2} p_{1}-q_{1} p_{2}\right)}{(\vec{q}-\vec{p})^{2}} e^{-z q^{2}}
$$

with only the $q$ integration left in (11); term-wise one gets (with $d \equiv x+z, b \equiv z+\mu$ and, $c \equiv d+\mu$ ):

1. $x \int_{0}^{\infty} d \mu d v e^{-(\mu+v+z) q^{2}+2 v \vec{q} \cdot \vec{p}-v p^{2}}\left(q_{2}-p_{2}\right)\left(q_{2}^{2}-q_{1}^{2}\right)\left(q_{2} p_{1}-q_{1} p_{2}\right)$

$$
\begin{aligned}
& =\frac{\pi x p_{1}}{2} e^{-z p^{2}} \int_{0}^{\infty} d \mu e^{-\mu p^{2}}\left\{\frac{\left(p_{1}^{2}-p_{2}^{2}\right)}{b^{2} p^{2}}+\frac{\left(p_{1}^{2}-3 p_{2}^{2}\right)}{b^{3} p^{4}}\left(2+e^{\frac{a}{b}}\right)+3 \frac{\left(p_{1}^{2}-3 p_{2}^{2}\right)}{b^{4} p^{6}}\left(1-e^{\frac{a}{b}}\right)\right\} \\
& =\frac{\pi x p_{1}}{2}\left\{\frac{e^{-z p^{2}}}{2 z}\left[1+\left(p_{1}^{2}-3 p_{2}^{2}\right)\left(\frac{1}{z p^{4}}+\frac{2}{z^{2} p^{6}}\right)\right]+\frac{\left(p_{1}^{2}-3 p_{2}^{2}\right)\left(\frac{1}{2}-z p^{2}\right)}{z^{2} p^{4}}-\frac{p^{2}}{2} \Gamma\left(0, z p^{2}\right)\right\} \equiv \frac{\pi p_{1}}{2} s_{1}
\end{aligned}
$$

2. $-2 x \int_{0}^{\infty} d \mu d v e^{-(\mu+v+x+z) q^{2}+2 v \vec{q} \cdot \vec{p}-v p^{2}}\left(q_{2}-p_{2}\right) q_{1}^{2}\left(q_{2} p_{1}-q_{1} p_{2}\right)$

$$
\begin{aligned}
& =-\frac{\pi x p_{1}}{2} e^{-(x+z) p^{2}} \int_{0}^{\infty} d \mu e^{-\mu p^{2}}\left\{-\frac{2 p_{1}^{2}}{c^{2} p^{2}}+\frac{1}{c^{3}}\left[\frac{4\left(p_{2}^{2}-p_{1}^{2}\right)}{p^{4}}+\frac{e^{\frac{b}{c}}}{p^{4}}\left(p^{2}+4 p_{2}^{2}\right)\right]+\frac{3\left(p_{1}^{2}-3 p_{2}^{2}\right)}{c^{4} p^{6}}\left(-1+e^{\frac{b}{c}}\right)\right\} \\
& =\frac{\pi x p_{1}}{4 d}\left[e^{-d p^{2}}\left\{1+\frac{p_{2}^{2}-3 p_{1}^{2}}{d p^{4}}+\frac{2\left(p_{1}^{2}-3 p_{2}^{2}\right)}{d^{2} p^{6}}\right\}-d p^{2} \Gamma\left(0, d p^{2}\right)+\frac{2}{d p^{2}}\left\{1+\frac{4 p_{2}^{2}}{p^{2}}+\frac{2}{d p^{4}}\left(p_{1}^{2}-3 p_{2}^{2}\right)\right\}\right] \\
& \equiv \frac{\pi p_{1}}{2} s_{2}
\end{aligned}
$$

3. $-2 \int_{0}^{\infty} \mu d \mu d v e^{-(\mu+v+z) q^{2}+2 v \vec{q} \cdot \vec{p}-v p^{2}}\left(q_{2}-p_{2}\right)\left(q_{2}^{2}-q_{1}^{2}\right)\left(q_{2} p_{1}-q_{1} p_{2}\right)$

$$
\begin{aligned}
& =-2 \frac{\pi p_{1}}{2} e^{-z p^{2}} \int_{0}^{\infty} \mu d \mu e^{-\mu p^{2}}\left\{\frac{\left(p_{1}^{2}-p_{2}^{2}\right)}{b^{2} p^{2}}+\frac{\left(p_{1}^{2}-3 p_{2}^{2}\right)}{b^{3} p^{4}}\left(2+e^{\frac{a}{b}}\right)+3 \frac{\left(p_{1}^{2}-3 p_{2}^{2}\right)}{b^{4} p^{6}}\left(1-e^{\frac{a}{b}}\right)\right\} \\
& =\frac{\pi p_{1}}{2}\left\{e^{-z p^{2}}\left[1-\frac{\left(p_{1}^{2}-3 p_{2}^{2}\right)}{z^{2} p^{6}}\right]-\left(1+z p^{2}\right) \Gamma\left(0, z p^{2}\right)-\frac{\left(p_{1}^{2}-3 p_{2}^{2}\right)}{z^{2} p^{6}}\left(1+z p^{2}\right)\right\} \equiv \frac{\pi p_{1}}{2} s_{3}
\end{aligned}
$$

4. $\quad 2 \int_{0}^{\infty} \mu d \mu d v e^{-(\mu+v+x+z) q^{2}+2 v \vec{q} \cdot \vec{p}-v p^{2}}\left(q_{2}-p_{2}\right)\left(q_{2}^{2}-q_{1}^{2}\right)\left(q_{2} p_{1}-q_{1} p_{2}\right)$

$$
\begin{aligned}
& =2 \frac{\pi p_{1}}{2} e^{-(x+z) p^{2}} \int_{0}^{\infty} \mu d \mu e^{-\mu p^{2}}\left\{\frac{\left(p_{1}^{2}-p_{2}^{2}\right)}{b^{2} p^{2}}+\frac{\left(p_{1}^{2}-3 p_{2}^{2}\right)}{b^{3} p^{4}}\left(2+e^{\frac{a}{b}}\right)+3 \frac{\left(p_{1}^{2}-3 p_{2}^{2}\right)}{b^{4} p^{6}}\left(1-e^{\frac{a}{b}}\right)\right\} \\
& =\frac{\pi p_{1}}{2}\left\{e^{-d p^{2}}\left[-1+\frac{\left(p_{1}^{2}-3 p_{2}^{2}\right)}{d^{2} p^{6}}\right]+\left(1+d p^{2}\right) \Gamma\left(0, d p^{2}\right)+\frac{\left(p_{1}^{2}-3 p_{2}^{2}\right)\left(1+d p^{2}\right)}{d^{2} p^{6}}\right\} \equiv \frac{\pi p_{1}}{2} s_{4}
\end{aligned}
$$

Thus

$$
K_{0}=-\frac{1}{2 \pi}\left(\frac{\lambda}{4 x}\right)^{3} p_{1}\left(s_{1}+s_{2}+s_{3}+s_{4}\right)
$$

with the $s_{i}$ defined by eqs. $(12 \mathrm{a}-\mathrm{d})$. Repeating the above exercise for $K_{2}(\vec{p})$ now written as

$$
K_{2}(\vec{p})=\left(-\frac{\lambda}{4 \pi}\right)^{3}(-2)^{2} \int p_{1} \delta(\vec{r}-\vec{s}) \frac{\left(p_{2}-r_{2}\right)\left(p_{2} r_{1}-p_{1} r_{2}\right)}{(\vec{p}-\vec{r})^{2}} e^{-x r^{2}} \frac{\left(s_{2}-q_{2}\right)\left(s_{2} q_{1}-s_{1} q_{2}\right)}{(\vec{s}-\vec{q})^{2}} e^{-z q^{2}}
$$

yields for the $s$ integration $\frac{\pi}{2 c^{2}} e^{-i\left(\alpha q_{1}+\beta q_{2}\right)}\left[\frac{q_{1}}{4}\left(\alpha^{2}-\beta^{2}\right)+\frac{\alpha \beta}{2} q_{2}\right]$ with $4 c=\alpha^{2}+\beta^{2}$; 
the $q$ integration now yields: $\left(\frac{\pi}{2}\right)^{2}\left(-\frac{i \alpha}{z^{2} c} e^{-\frac{c}{z}}\right)$; and the $\alpha, \beta$ integration $\left(\frac{\pi}{2}\right)^{3} \frac{16 r_{1}}{r^{2} z^{2}}\left(1-e^{-z r^{2}}\right)$ leaving only the $\mathrm{r}$ - integration the relevant integral for which is

$$
16\left(\frac{\pi}{2}\right)^{3} \frac{1}{z^{2}} \int r_{1} \frac{\left(p_{2}-r_{2}\right)\left(p_{2} r_{1}-p_{1} r_{2}\right)}{r^{2}(\vec{p}-\vec{r})^{2}} e^{-x r^{2}}\left(1-e^{-z r^{2}}\right)
$$

One finally gets with $d \equiv(x+z)$ :

$$
\begin{aligned}
& K_{2}=-\frac{z}{\pi}\left(\frac{\lambda}{4 z}\right)^{3} p_{1}\left[\left\{-\frac{e^{-x p^{2}}}{2 x}\left(1+\frac{\left(p_{1}^{2}-p_{2}^{2}\right)}{x p^{4}}\right)+\frac{1}{x p^{2}}\left[p_{2}^{2}+\frac{\left(p_{1}^{2}-p_{2}^{2}\right)}{2 x p^{4}}\right]+\frac{p^{2}}{2} \Gamma\left(0, z p^{2}\right)\right\}-\left\{-\frac{e^{-d p^{2}}}{2 d}(1+\right.\right. \\
& \left.\left.\left.\frac{\left(p_{1}^{2}-p_{2}^{2}\right)}{d p^{4}}\right)+\frac{1}{d p^{2}}\left[p_{2}^{2}+\frac{\left(p_{1}^{2}-p_{2}^{2}\right)}{2 d p^{4}}\right]+\frac{p^{2}}{2} \Gamma\left(0, d p^{2}\right)\right\}\right]
\end{aligned}
$$

The remaining integral $K_{3}$ is too cumbersome to work out below; therefore its calculation will only be sketched here. By writing it as

$K_{3}=\left(\frac{\lambda}{2 \pi}\right)^{3} \int \delta(\vec{r}-\vec{s}) \delta(\vec{t}-\vec{q}) e^{-x r^{2}} \frac{\left(p_{2}-r_{2}\right)\left(p_{2} r_{1}-p_{1} r_{2}\right)}{(\vec{p}-\vec{r})^{2}} \frac{\left(s_{2}-t_{2}\right)\left(s_{2} t_{1}-s_{1} t_{2}\right)}{(\vec{s}-\vec{t})^{2}} \frac{\left(q_{2}-p_{2}\right)\left(q_{2} p_{1}-q_{1} p_{2}\right)}{(\vec{q}-\vec{p})^{2}} e^{-z q^{2}}$

with $(\vec{r}-\vec{s})=\left(\frac{1}{2 \pi}\right)^{2} \iint_{-\infty}^{\infty} d \alpha d \beta e^{i \alpha\left(r_{1}-s_{1}\right)+i \beta\left(r_{2}-s_{2}\right)}, \delta(\vec{t}-\vec{q})=\left(\frac{1}{2 \pi}\right)^{2} \iint_{-\infty}^{\infty} d v d \theta e^{i \theta\left(t_{1}-q_{1}\right)+i v\left(t_{2}-q_{2}\right)}$ the $s$ integration leads to $\frac{\pi}{2 c^{2}} e^{-i\left(\alpha t_{1}+\beta t_{2}\right)}\left(\frac{1}{4}\left(\alpha^{2}-\beta^{2}\right) t_{1}+\frac{1}{2} \alpha \beta t_{2}\right), 4 c \equiv \alpha^{2}+\beta^{2}$.Integrating over $\alpha, \beta$ gives

$\frac{2 \pi^{2}}{h^{2}}\left\{h_{2}\left(r_{2} t_{1}-r_{1} t_{2}\right)-h_{1}\left(h_{1} t_{1}+h_{2} t_{2}\right)\right\}$

with $\vec{h}=\vec{r}-\vec{t}$; and the $t$ integration yields $\frac{\pi^{3}}{2 g^{2}} e^{i\left(\theta r_{1}+v r_{2}\right)}\left[\left(\theta^{2}-v^{2}\right) r_{1}+2 \theta v r_{2}\right], 4 g=\theta^{2}+v^{2}$; the $\theta, v$ integration can now be done to get with $\vec{b}=\vec{r}-\vec{q}: \frac{8 \pi^{4}}{b^{2}}\left\{b_{2}\left[r_{2} q_{1}-r_{1} q_{2}\right]-r_{1}\left[r_{1} b_{1}+r_{2} b_{2}\right]\right\}$

Only the $r$ and $q$ integration now remain; to take up the latter first we have with $\vec{m}=\alpha \vec{r}+\beta \vec{p}$, $j \equiv \alpha+l, l=\beta+z, k=\alpha+z, g \equiv[\alpha(\vec{r}-\vec{p})-z \vec{p}]^{2}$ and $n=[\beta(\vec{p}-\vec{r})-z \vec{r}]^{2}$ :

$8 \pi^{4} \int d^{2} q \frac{\left(q_{2}-p_{2}\right)\left(q_{2} p_{1}-q_{1} p_{2}\right)}{b^{2}(\vec{q}-\vec{p})^{2}} e^{-z q^{2}}\left\{b_{2}\left[r_{2} q_{1}-r_{1} q_{2}\right]-r_{1}\left[r_{1} b_{1}+r_{2} b_{2}\right]\right\}$ $=\pi^{5} e^{-z r^{2}} \int_{0}^{\infty} d \alpha d \beta\left\{e^{-\beta(\vec{p}-\vec{r})^{2}} \frac{e^{\frac{n}{j}}}{j^{7}}\left\{-8 \alpha \beta j^{2} m_{2}^{2}\left(p_{1} r_{2}-p_{2} r_{1}\right)^{2}+4 j^{3}\left[r_{2} p_{2}\left(m_{1}^{2}+m_{2}^{2}\right)+3 m_{2}\left(\beta p_{1}-\right.\right.\right.\right.$ $\left.\left.\alpha r_{1}\right)\left(r_{1} p_{2}-r_{2} p_{1}\right)+2 \alpha \beta m_{2}\left(r_{2}+p_{2}\right)\left(p_{1} r_{2}-p_{2} r_{1}\right)^{2}\right]+2 j^{4}\left[\left(r_{2} p_{2}+3 r_{1} p_{1}\right)+2\left(r_{2}+p_{2}\right)\left(\beta p_{1}-\right.\right.$ $\left.\left.\alpha r_{1}\right)\left(p_{1} r_{2}-p_{2} r_{1}\right)-2 m_{2}\left(r_{2}+p_{2}\right)\left(r_{2} p_{2}+r_{1} p_{1}\right)-4 \alpha \beta r_{2} p_{2}\left(p_{1} r_{2}-p_{2} r_{1}\right)^{2}\right]+4 j^{5} r_{2} p_{2}\left(r_{2} p_{2}+\right.$ $\left.\left.r_{1} p_{1}\right)\right\}-2 r_{1} e^{-\alpha(\vec{p}-\vec{r})^{2}} \frac{e^{\frac{g}{j}}}{j^{6}}\left\{4 \alpha j^{2} m_{2}\left[\left(r_{1} p_{2}-r_{2} p_{1}\right)\left(m_{1} r_{1}+m_{2} r_{2}\right)\right]+2 j^{3}\left[\left(r_{1} p_{2}-r_{2} p_{1}\right)\left(m_{2}+\alpha r_{2}\right)-\right.\right.$ $\left.p_{1}\left(m_{1} r_{1}+m_{2} r_{2}\right)-2 \alpha\left(r_{1} p_{2}-r_{2} p_{1}\right)\left\{m_{2} r^{2}+p_{2}\left(m_{1} r_{1}+m_{2} r_{2}\right)\right\}\right]+2 j^{4}\left[r^{2}\left(p_{1}+2 \alpha p_{2}\left(r_{1} p_{2}-\right.\right.\right.$ 
$\left.\left.\left.\left.\left.r_{2} p_{1}\right)\right)-p_{2}\left(r_{1} p_{2}-r_{2} p_{1}\right)\right]\right\}\right\}$

There now remains the integration over $r$ and $\alpha($ or $\beta)$ on each term in (17); this will be presented elsewhere.

\section{Summary}

The calculation of both $K_{0}$ and $K_{2}$ in the preceding section has been tedious but has been catalyzed using the Dirac $\delta$-function and its integral representation; its workout in detail was motivated by the simplicity of the method and also because to the best of our knowledge this has not been used elsewhere.

Acknowledgements: I thank the Indian Institute of Technology Madras for their financial support and encouragement in enabling me to attend and present this work at the $36^{\text {th }}$ ICHEP2012 at Melbourne.

\section{References}

[1] See for example N.Piskunov, Differential and Integral Calculus, Mir Publishers, Moscow(1974).

[2] S.G.Kamath, Reworking the Antonsen-Bormann idea, J.Phys.Conf.Ser.343(2012)12051 ed.by Čestmír Burdík, Ondřej Navrátil, Severin Pošta, Martin Schnabl and Libor Šnobl .

[3] http://functions.wolfram.com/

[4] Gopinath Kamath, Reworking the Antonsen-Bormann idea I., AIP Conf.Proc.1446(2010)201 ed.by J.Kouneiher, C.Barbachoux, T.Masson and D.Vey.

[5] D.G.C.McKeon and T.N.Sherry, "Operator regularization and one loop Green's functions", Phys.Rev.D35,3854(1987)

[6] J.Schwinger, "On gauge invariance and vacuum polarization", Phys.Rev.82,664(1951) 